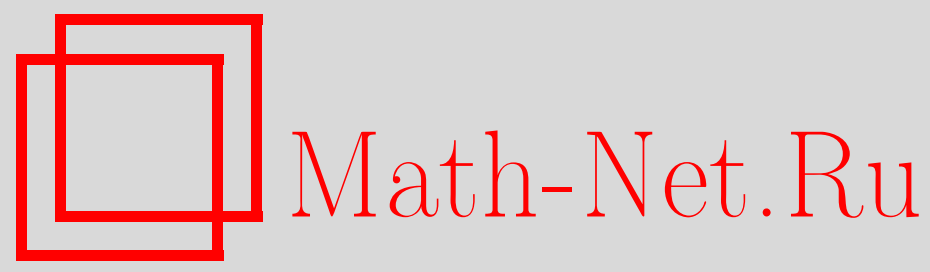

Р. А. Алиев, О представимости аналитических функций по своим граничным значениям, Матем. заметки, 2003, том 73 , выпуск 1, 8-21

DOI: https://doi.org/10.4213/mzm164

Использование Общероссийского математического портала Math-Net.Ru подразумевает, что вы прочитали и согласны с пользовательским соглашением http://www.mathnet.ru/rus/agreement

Параметры загрузки:

IP : 54.237 .59 .107

26 апреля 2023 г., 18:07:49

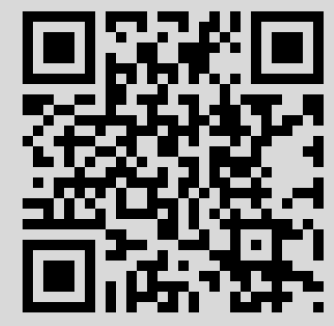




\section{О ПРЕДСТАВИМОСТИ АНАЛИТИЧЕСКИХ ФУНКЦИЙ ПО СВОИМ ГРАНИЧНЫМ ЗНАЧЕНИЯМ}

\section{Р. А. Алиев}

Пусть $\nu$ - произвольная конечная комплексная борелевская мера на интервале [0; $2 \pi), u\left(r e^{i \varphi}\right)$ - ее интеграл Пуассона, а $v\left(r e^{i \varphi}\right)$ - функция, гармонически сопряженная с $u\left(r e^{i \varphi}\right), F(z)=u(z)+i v(z), z=r e^{i \varphi}, F(t)$ - некасательное предельне значение аналитической функции $F(z)$ при $z \rightarrow t=e^{i \theta}$. В работе рассматривается вопрос о представлении аналитической функции $F(z)$ через граничные значения $F(t)$.

Библиограффия: 9 названий.

В своей заметке П. Л. Ульянов [1] показал, что если $\nu$ - абсолютно непрерьвная конечная мера относительно меры Лебега на интервале $[0 ; 2 \pi), u\left(r e^{i \varphi}\right)$ - ее интеграл Пуaccoна, а $v\left(r e^{i \varphi}\right)$ - функция, гармонически сопряженная с $u\left(r e^{i \varphi}\right)$, то аналитическую функцию $F(z)=u(z)+i v(z), z=r e^{i \varphi}$, можно представить $A$-интегралом Коши от своих граничных значений, т.е. справедлива формула

$$
F(z)=\frac{1}{2 \pi i} \lim _{\lambda \rightarrow+\infty} \int_{T_{\lambda}} \frac{F(t)}{t-z} d t, \quad z=r e^{i \varphi},
$$

где $F(t)$ - некасательное предельное значение аналитической функции $F(z)$ при $z \rightarrow$ $t=e^{i \theta}$ и $T_{\lambda}=\left\{t=e^{i \theta}:|F(t) /(t-z)| \leqslant \lambda\right\}$.

В дальнейшем И. А. Виноградовой [2], [3] было введено понятие $L G^{*}$-интеграла и доказано, что если функция $F(z)$ представима $L G^{*}$-интегралом типа Коши, то $F(z)$ представима $L G^{*}$-интегралом Коши. Так как $L G^{*}$-интеграл является сужением $A$-интеграла, формула (1) остается в силе и в этом случае.

Однако когда мера $\nu$ не является абсолютно непрерывной, формула (1) становится несправедливой. Например, для дискретной меры

$$
\nu(X)= \begin{cases}2 \pi & \text { при } 0 \in X, \\ 0 & \text { при } 0 \notin X,\end{cases}
$$

аналитическая функция $F(z)=u(z)+i v(z)$ и ее граничные значения соответственно будут $F(z)=(1+z) /(1-z)$ и $F(t)=(1+t) /(1-t)$, но предел

$$
\begin{aligned}
\frac{1}{2 \pi i} \lim _{\lambda \rightarrow+\infty} \int_{T_{\lambda}} \frac{F(t)}{t-z} d t & =\mathrm{v} \cdot \mathrm{p} \cdot \frac{1}{2 \pi i} \int_{T} \frac{1+t}{1-t} \cdot \frac{1}{t-z} d t \\
& =\mathrm{v} \cdot \mathrm{p} \cdot \frac{1}{2 \pi i} \int_{T} \frac{1+t}{1-z}\left\{\frac{1}{1-t}+\frac{1}{t-z}\right\} d t
\end{aligned}
$$




$$
\begin{aligned}
& =\frac{1}{1-z} \mathrm{v} \cdot \mathrm{p} \cdot \frac{1}{2 \pi i} \int_{T} \frac{1+t}{1-t} d t+\frac{1}{1-z} \frac{1}{2 \pi i} \int_{T} \frac{1+t}{t-z} d t \\
& =\frac{z}{1-z} \neq F(z),
\end{aligned}
$$

где $T$ - единичная окружность в комплексной плоскости $C$ с центром в начале координат.

В настоящей работе рассматривается вопрос о представлении аналитической функции $F(z)=u(z)+i v(z)$ через граничные значения $F(t)$ для произвольных конечнњх комплексных мер.

Приведем некоторые определения и обозначения, используемые в дальнейшем.

Через $M_{C}, M_{R}, M_{+}, M_{S}$ будем обозначать соответственно множество всех конечных комплексных, действительных, неотрицательных и сингулярных борелевских мер на интервале $T_{0}=[0 ; 2 \pi)$. Будем говорить, что мера $\nu$ сосредоточена на борелевском множестве $A \subset T_{0}$, если для всех борелевских множеств $B \subset T_{0} \nu(B)=\nu(B \cap A)$. Через $\operatorname{supp} \nu$ будем обозначать носитель меры $\nu$ (т.е. наименьшее замкнутое множество $E \subset T_{0}$, на котором сосредоточена мера $\nu$ ), а через $\|\nu\|$ - полную вариацию этой меры.

Для любой действительной функции $f$ на $T_{0}$ обозначим

$$
\begin{array}{ll}
(f>\lambda) \stackrel{\text { def }}{=}\left\{\theta \in T_{0}: f(\theta)>\lambda\right\}, & (f<\lambda) \stackrel{\text { def }}{=}\left\{\theta \in T_{0}: f(\theta)<\lambda\right\}, \\
(f \geqslant \lambda) \stackrel{\text { def }}{=}\left\{\theta \in T_{0}: f(\theta) \geqslant \lambda\right\}, & (f \leqslant \lambda) \stackrel{\text { def }}{=}\left\{\theta \in T_{0}: f(\theta) \leqslant \lambda\right\} .
\end{array}
$$

1. Равенство Рисса для конечных комплексных мер. Пусть $\nu \in M_{C}$. Функция $\tilde{\nu}$, сопряженная к мере $\nu$, определяется следующим образом:

$$
\tilde{\nu}(\theta)=\frac{1}{2 \pi} \lim _{\varepsilon \rightarrow 0+} \int_{\left\{t \in T_{0}:|t-\theta|>\varepsilon\right\}} \operatorname{ctg} \frac{\theta-t}{2} d \nu(t), \quad \theta \in T_{0} ;
$$

в частности, когда $\nu$ абсолютно непрерьвна, $d \nu(t)=f(t) d t$, то функция $\tilde{\nu}$ называется также сопряженной к функции $f(t)$ и обозначается через $\bar{f}$.

Теорема М. Рисса [4] утверждает, что если мера $\nu$ абсолютно непрерьвна, $d \nu(t)=$ $f(t) d t$ и функция $f(t) \in L^{p}[0 ; 2 \pi), 1<p<+\infty$, то для любой функции $g(t) \in L^{q}[0 ; 2 \pi)$, где $1 / p+1 / q=1$, имеет место равенство

$$
\int_{0}^{2 \pi} \bar{g}(\theta) d \nu(\theta)=-\lim _{\lambda \rightarrow+\infty} \int_{(|\tilde{\nu} \cdot g| \leqslant \lambda)} \tilde{\nu}(\theta) g(\theta) d \theta .
$$

В дальнейшем Ульяновым [5] было доказано, что равенство (2) остается в силе, когда $f(t) \in L[0 ; 2 \pi)$ и $g(t)$ такая ограниченная функция, что $\bar{g}(t)$ также является ограниченной функцией.

В этом пункте доказывается

Теорема 1. Если $\nu$ - конечная комплексная борелевская мера на $T_{0}$ и функиия $g(t)$ непрерывна по Гёльдеру на $T_{0}$, то существует предел

$$
\lim _{\lambda \rightarrow+\infty} \int_{(|\tilde{\nu} \cdot g| \leqslant \lambda \mid)} \tilde{\nu}(\theta) g(\theta) d \theta
$$

и справедливо равенство (2).

Мы будем использовать следующие две теоремы, доказанные С.А. Виноградовым, С. В. Хрущевым [6] (теорема А) и Т. С. Салимовым [7] (теорема В). 
Tеорема А [6]. Для любой конечной комплексной борелевской меры $\mu$ на единичной окружности $T$ комплексной плоскости $C$ справедливо равенство

$$
\lim _{\lambda \rightarrow+\infty} \lambda \cdot m\left\{\theta \in T_{0}:\left|\tilde{\mu}\left(e^{i \theta}\right)\right|>\lambda\right\}=\frac{2}{\pi}\left\|\mu_{s}\right\|,
$$

где $\mu_{s}$ - сингулярная часть меры $\mu, m$ - мера Лебега.

Теорема В [7]. Для любой конечной комплексной борелевской меры $\mu$ на Т выполняется равенство

$$
\lim _{\lambda \rightarrow+\infty} \int_{\left\{\theta \in T_{0}:\left|\tilde{\mu}\left(e^{i \theta}\right)\right| \leqslant \lambda\right\}} \tilde{\mu}\left(e^{i \theta}\right) d \theta=0 .
$$

ДокАЗАТЕЛЬСТво ТЕОРЕМЫ 1. Так как

$$
\begin{aligned}
\bar{g}(t) & =\frac{1}{2 \pi} \lim _{\varepsilon \rightarrow 0+} \int_{\{\theta:|\theta-t|>\varepsilon\}} \operatorname{ctg} \frac{t-\theta}{2} g(\theta) d \theta \\
& =\frac{1}{2 \pi} \int_{0}^{2 \pi} \operatorname{ctg} \frac{t-\theta}{2}[g(\theta)-g(t)] d \theta+\frac{1}{2 \pi} g(t) \lim _{\varepsilon \rightarrow 0+} \int_{\{\theta:|\theta-t|>\varepsilon\}} \operatorname{ctg} \frac{t-\theta}{2} d \theta \\
& =\frac{1}{2 \pi} \int_{0}^{2 \pi} \operatorname{ctg} \frac{\theta-t}{2}[g(t)-g(\theta)] d \theta
\end{aligned}
$$

и функция

$$
K(t, \theta)=\operatorname{ctg} \frac{\theta-t}{2}[g(t)-g(\theta)]
$$

абсолютно интегрируема по $\theta$ и существует интеграл

$$
\int_{0}^{2 \pi}\left(\int_{0}^{2 \pi}|K(t, \theta)| d \theta\right) d \nu(t)
$$

то (см. $[8$, гл. $5, \S 6])$

$$
\begin{aligned}
\int_{0}^{2 \pi} \bar{g}(t) d \nu(t) & =\frac{1}{2 \pi} \int_{0}^{2 \pi}\left(\int_{0}^{2 \pi} \operatorname{ctg} \frac{\theta-t}{2}[g(t)-g(\theta)] d \theta\right) d \nu(t) \\
& =\frac{1}{2 \pi} \int_{0}^{2 \pi}\left(\int_{0}^{2 \pi} \operatorname{ctg} \frac{\theta-t}{2}[g(t)-g(\theta)] d \nu(t)\right) d \theta .
\end{aligned}
$$

Рассмотрим меру $d \mu\left(e^{i \theta}\right)=g(\theta) d \nu(\theta), \theta \in[0 ; 2 \pi)$, на единичной окружности $T$. По теореме B

$$
\lim _{\lambda \rightarrow+\infty} \int_{\left\{\theta \in T_{0}:\left|\tilde{\mu}\left(e^{i \theta}\right)\right| \leqslant \lambda\right\}} \tilde{\mu}\left(e^{i \theta}\right) d \theta=0,
$$

где

$$
\begin{aligned}
\tilde{\mu}\left(e^{i \theta}\right) & =\frac{1}{2 \pi} \lim _{\varepsilon \rightarrow 0+} \int_{\left\{t \in T_{0}:|t-\theta|>\varepsilon\right\}} \operatorname{ctg} \frac{\theta-t}{2} d \mu\left(e^{i t}\right) \\
& =\frac{1}{2 \pi} \lim _{\varepsilon \rightarrow 0+} \int_{\left\{t \in T_{0}:|t-\theta|>\varepsilon\right\}} \operatorname{ctg} \frac{\theta-t}{2} g(t) d \nu(t) \\
& =\frac{1}{2 \pi} \int_{0}^{2 \pi} \operatorname{ctg} \frac{\theta-t}{2}[g(t)-g(\theta)] d \nu(t)+g(\theta) \tilde{\nu}(\theta) .
\end{aligned}
$$


Из равенства (3) следует, что функция

$$
J(\theta)=\frac{1}{2 \pi} \int_{0}^{2 \pi} \operatorname{ctg} \frac{\theta-t}{2}[g(t)-g(\theta)] d \nu(t)
$$

интегрируема на интервале $[0 ; 2 \pi)$. Тогда в силу (4) из (5) получим, что

$$
\int_{0}^{2 \pi} J(\theta) d \theta+\lim _{\lambda \rightarrow+\infty} \int_{\left\{\theta \in T_{0}:\left|\tilde{\mu}\left(e^{i \theta}\right)\right| \leqslant \lambda\right\}} g(\theta) \tilde{\nu}(\theta) d \theta=0
$$

Это означает, что существует предел

$$
\lim _{\lambda \rightarrow+\infty} \int_{\left\{\theta \in T_{0}:\left|\tilde{\mu}\left(e^{i \theta}\right)\right| \leqslant \lambda\right\}} g(\theta) \tilde{\nu}(\theta) d \theta
$$

и справедливо равенство

$$
\lim _{\lambda \rightarrow+\infty} \int_{\left\{\theta \in T_{0}:\left|\tilde{\mu}\left(e^{i \theta}\right)\right| \leqslant \lambda\right\}} g(\theta) \tilde{\nu}(\theta) d \theta=-\int_{0}^{2 \pi} J(\theta) d \theta=-\int_{0}^{2 \pi} \bar{g}(t) d \nu(t) .
$$

Из интегрируемости функции $J(\theta)$ вытекает, что

$$
m\{\theta:|J(\theta)| \geqslant \lambda\}=o\left(\frac{1}{\lambda}\right), \quad \lambda \rightarrow+\infty
$$

Так как $\tilde{\mu}\left(e^{i \theta}\right)=J(\theta)+g(\theta) \tilde{\nu}(\theta)$ и по теореме А существует конечньй предел

$$
\lim _{\lambda \rightarrow+\infty} \lambda m(|\tilde{\mu}|>\lambda)=\frac{2}{\pi}\left\|\mu_{s}\right\|
$$

то

$$
\begin{aligned}
m((|\tilde{\mu}|>\lambda) \cap(|g \tilde{\nu}| \leqslant \lambda)) & \leqslant m(\lambda<|\tilde{\mu}| \leqslant(1+\alpha) \lambda)+m((|\tilde{\mu}|>(1+\alpha) \lambda) \cap(|g \tilde{\nu}| \leqslant \lambda)) \\
& \leqslant m(|\tilde{\mu}|>\lambda)-m(|\tilde{\mu}|>(1+\alpha) \lambda)+m(|J|>\alpha \lambda) .
\end{aligned}
$$

Отсюда

$$
\lim _{\lambda \rightarrow+\infty} \lambda m((|\tilde{\mu}|>\lambda) \cap(|g \tilde{\nu}| \leqslant \lambda)) \leqslant \frac{2}{\pi} \frac{\alpha}{1+\alpha}\left\|\mu_{s}\right\| .
$$

Из произвольности $\alpha$ получим, что

$$
\lim _{\lambda \rightarrow+\infty} \lambda m((|\tilde{\mu}|>\lambda) \cap(|g \tilde{\nu}| \leqslant \lambda))=0 .
$$

Аналогично доказывается, что

$$
\lim _{\lambda \rightarrow+\infty} \lambda m((|\tilde{\mu}| \leqslant \lambda) \cap(|g \tilde{\nu}|>\lambda))=0 .
$$


Тогда

$$
\begin{aligned}
\int_{(|\tilde{\nu} g| \leqslant \lambda)} \tilde{\nu}(\theta) g(\theta) d \theta= & \int_{(|\tilde{\mu}| \leqslant \lambda)} \tilde{\nu}(\theta) g(\theta) d \theta+\int_{(|\tilde{\nu} g| \leqslant \lambda) \cap(|\tilde{\mu}|>\lambda)} \tilde{\nu}(\theta) g(\theta) d \theta \\
& -\int_{(|\tilde{\mu}| \leqslant \lambda) \cap(|\tilde{\nu} g|>\lambda)} \tilde{\nu}(\theta) g(\theta) d \theta=I_{1}+I_{2}+I_{3} .
\end{aligned}
$$

Поскольку $\left|I_{2}\right| \leqslant \lambda m((|g \tilde{\nu}| \leqslant \lambda) \cap(|\tilde{\mu}|>\lambda)) \rightarrow 0$ при $\lambda \rightarrow \infty$ и

$$
\begin{aligned}
\left|I_{3}\right| & =\left|\int_{(|\tilde{\mu}| \leqslant \lambda) \cap(|\tilde{\nu} g|>\lambda)}\left(\tilde{\mu}\left(e^{i \theta}\right)-J(\theta)\right) d \theta\right| \\
& \leqslant \lambda m((|\tilde{\mu}| \leqslant \lambda) \cap(|\tilde{\nu} g|>\lambda))+\int_{(|\tilde{\mu}| \leqslant \lambda) \cap(|\tilde{\nu} g|>\lambda)}|J(\theta)| d \theta \rightarrow 0 \quad \text { при } \lambda \rightarrow \infty,
\end{aligned}
$$

из существования предела

$$
\lim _{\lambda \rightarrow+\infty} \int_{\left\{\theta \in T_{0}:\left|\tilde{\mu}\left(e^{i \theta}\right)\right| \leqslant \lambda\right\}} g(\theta) \tilde{\nu}(\theta) d \theta
$$

следует существование предела

$$
\lim _{\lambda \rightarrow+\infty} \int_{(|\tilde{\nu} \cdot g| \leqslant \lambda \mid)} \tilde{\nu}(\theta) g(\theta) d \theta
$$

и их равенство, а из равенства (6) следует равенство (2). Теорема доказана.

2. Представимость аналитических функций по своим граничным значениям. Для произвольных конечных мер сначала докажем следующую теорему.

Теорема 2. Пусть мера $\nu \in M_{C}, u\left(r e^{i \varphi}\right)$ - ее интеграл Пуассона, а $v\left(r e^{i \varphi}\right)$ функиия, гармонически сопряженная с и(re $\left.\mathrm{i}^{i \varphi}\right)$. Тогда

$$
F(z)=\frac{1}{2 \pi i} \lim _{\lambda \rightarrow+\infty} \int_{T_{\lambda}} \frac{F(t)}{t-z} d t+\frac{1}{2 \pi} \int_{0}^{2 \pi} \frac{e^{i \theta}}{e^{i \theta}-z} d \nu_{s}(\theta), \quad z=r e^{i \varphi}
$$

где $F(z)=u(z)+i v(z), F(t)$ - некасательное предельное значение функиии $F(z)$ при $z \rightarrow t=e^{i \theta}, T_{\lambda}=\left\{t=e^{i \theta}:|F(t) /(t-z)| \leqslant \lambda\right\}$ и $\nu_{s}-$ сингулярная часть меры $\nu$.

ДоказАТЕЛьство. Так как

$$
u(z)=\frac{1}{2 \pi} \int_{0}^{2 \pi} P_{z}(\theta) d \nu(\theta), \quad v(z)=-\frac{1}{2 \pi} \int_{0}^{2 \pi} Q_{z}(\theta) d \nu(\theta)
$$

где

$$
\begin{aligned}
P_{z}(\theta) & =\operatorname{Re} \frac{e^{i \theta}+z}{e^{i \theta}-z}=\frac{1-r^{2}}{1-2 r \cos (\theta-\varphi)+r^{2}}, \quad z=r e^{i \varphi}, \\
Q_{z}(\theta) & =-\operatorname{Im} \frac{e^{i \theta}+z}{e^{i \theta}-z}=\frac{2 r \sin (\theta-\varphi)}{1-2 r \cos (\theta-\varphi)+r^{2}},
\end{aligned}
$$


то

$$
\begin{aligned}
F(z) & =u(z)+i v(z)=\frac{1}{2 \pi} \int_{0}^{2 \pi} P_{z}(\theta) d \nu(\theta)-\frac{i}{2 \pi} \int_{0}^{2 \pi} Q_{z}(\theta) d \nu(\theta) \\
& =\frac{1}{2 \pi} \int_{0}^{2 \pi}\left[P_{z}(\theta)-\frac{r e^{i(\theta-\varphi)}}{1-r e^{i(\theta-\varphi)}}\right] d \nu(\theta)-\frac{i}{2 \pi} \int_{0}^{2 \pi}\left[Q_{z}(\theta)+\frac{i r e^{i(\theta-\varphi)}}{1-r e^{i(\theta-\varphi)}}\right] d \nu(\theta) .
\end{aligned}
$$

Обозначим

$$
R_{z}(\theta)=P_{z}(\theta)-\frac{r e^{i(\theta-\varphi)}}{1-r e^{i(\theta-\varphi)}} .
$$

Из доказанного в [1] равенства

$$
\overline{R_{z}}(\theta)=Q_{z}(\theta)+\frac{i r e^{i(\theta-\varphi)}}{1-r e^{i(\theta-\varphi)}}
$$

и из теоремы 1 получим, что

$$
F(z)=\frac{1}{2 \pi} \int_{0}^{2 \pi} R_{z}(\theta) d \nu(\theta)+\frac{i}{2 \pi} \lim _{\lambda \rightarrow+\infty} \int_{T_{\lambda}^{*}} R_{z}(\theta) \tilde{\nu}(\theta) d \theta
$$

где $T_{\lambda}^{*}=\left\{\theta \in T_{0}:\left|R_{z}(\theta) \tilde{\nu}(\theta)\right| \leqslant \lambda\right\}$.

$\mathrm{C}$ другой стороны

$$
R_{z}(\theta)=\frac{1}{1-r e^{i(\varphi-\theta)}}=\frac{e^{i \theta}}{e^{i \theta}-r e^{i \varphi}}
$$

так Kak

$$
\frac{1-r^{2}}{1-2 r \cos \alpha+r^{2}}-\frac{r e^{i \alpha}}{1-r e^{i \alpha}}=\frac{1}{1-r e^{-i \alpha}} .
$$

Полагая в формуле (8) $e^{i \theta}=t$, имеем

$$
F(z)=\frac{1}{2 \pi} \int_{0}^{2 \pi} \frac{e^{i \theta} d \nu(\theta)}{t-z}+\frac{i}{2 \pi} \lim _{\lambda \rightarrow+\infty} \int_{T_{\lambda}^{*}} \frac{e^{i \theta} \tilde{\nu}(\theta) d \theta}{t-z}
$$

и $T_{\lambda}^{*}=\left\{\theta \in T_{0}:|\tilde{\nu}(\theta) /(t-z)| \leqslant \lambda\right\}$.

Пусть $d \nu(\theta)=f(\theta) d \theta+d \nu_{s}(\theta)$. Тогда [9]

$$
F(t)=F\left(e^{i \theta}\right)=f(\theta)+i \tilde{\nu}(\theta) .
$$

Поэтому

$$
\begin{aligned}
F(z) & =\frac{1}{2 \pi} \int_{0}^{2 \pi} \frac{e^{i \theta} f(\theta) d \theta}{t-z}+\frac{1}{2 \pi} \int_{0}^{2 \pi} \frac{e^{i \theta} d \nu_{s}(\theta)}{t-z}+\frac{i}{2 \pi} \lim _{\lambda \rightarrow+\infty} \int_{T_{\lambda}^{*}} \frac{e^{i \theta} \tilde{\nu}(\theta) d \theta}{t-z} \\
& =\frac{1}{2 \pi} \int_{0}^{2 \pi} \frac{e^{i \theta}}{e^{i \theta}-z} d \nu_{s}(\theta)+\frac{1}{2 \pi i} \lim _{\lambda \rightarrow+\infty} \int_{T_{\lambda}^{*}} \frac{(f(\theta)+i \tilde{\nu}(\theta))}{t-z} i e^{i \theta} d \theta \\
& =\frac{1}{2 \pi} \int_{0}^{2 \pi} \frac{e^{i \theta}}{e^{i \theta}-z} d \nu_{s}(\theta)+\frac{1}{2 \pi i} \lim _{\lambda \rightarrow+\infty} \int_{T_{\lambda}^{*}} \frac{F(t)}{t-z} i e^{i \theta} d \theta .
\end{aligned}
$$


Из соотношения $m\left\{\theta \in T_{0}:|f(\theta) /(t-z)| \geqslant \lambda\right\}=o(1 / \lambda), \lambda \rightarrow+\infty$, вытекает, что $m\left(T_{\lambda} \Delta T_{\lambda}^{*}\right)=o(1 / \lambda), \lambda \rightarrow+\infty$.

Следовательно, из (9) получим, что

$$
F(z)=\frac{1}{2 \pi} \int_{0}^{2 \pi} \frac{e^{i \theta}}{e^{i \theta}-z} d \nu_{s}(\theta)+\frac{1}{2 \pi i} \lim _{\lambda \rightarrow+\infty} \int_{T_{\lambda}} \frac{F(t)}{t-z} d t
$$

Теорема доказана.

В дальнейшем функции, определенные на $T_{0}$, будем рассматривать как периодические функции, определенные на действительной прямой $\mathbb{R}$ с периодом $2 \pi$.

Для любой действительной функции $f$ на $T_{0}$ обозначим

$$
P\left(f ; q ; \theta_{0} ; \theta\right)=\frac{\pi}{2} \operatorname{ctg} \frac{\pi q^{n-1}}{2} \cdot f(\theta)
$$

при $\theta \in\left(\theta_{0}+\pi q^{n} ; \theta_{0}+\pi q^{n-1}\right) \cup\left(\theta_{0}-\pi q^{n-1} ; \theta_{0}-\pi q^{n}\right), n \in \mathbb{N}, 0<q<1, \theta_{0} \in T_{0}$,

$$
\begin{gathered}
P_{1}\left(f ; \theta_{0}\right)=\lim _{q \rightarrow 1-\alpha \rightarrow+\infty} \alpha \cdot m\left\{\theta \in\left(\theta_{0} ; \theta_{0}+\pi\right):\left|P\left(f ; q ; \theta_{0} ; \theta\right)\right|>\alpha\right\}, \\
P_{2}\left(f ; \theta_{0}\right)=\lim _{q \rightarrow 1-\alpha \rightarrow+\infty} \alpha \cdot m\left\{\theta \in\left(\theta_{0}-\pi ; \theta_{0}\right):\left|P\left(f ; q ; \theta_{0} ; \theta\right)\right|>\alpha\right\}, \\
r_{\lambda, f}\left(\theta_{0}\right)= \begin{cases}\operatorname{sign}\left(P_{2}\left(f ; \theta_{0}\right)-P_{1}\left(f ; \theta_{0}\right)\right) & \text { при } f\left(\theta_{0}\right)>\lambda, \\
0 & \text { при }\left|f\left(\theta_{0}\right)\right| \leqslant \lambda, \\
\operatorname{sign}\left(P_{1}\left(f ; \theta_{0}\right)-P_{2}\left(f ; \theta_{0}\right)\right) & \text { при } f\left(\theta_{0}\right)<-\lambda,\end{cases}
\end{gathered}
$$

если существуют пределы в правых частях равенств $(10),(11)$ почти для всех $\theta_{0} \in[0,2 \pi)$.

Отметим, что для любой меры $\nu \in M_{R}$ функция $r_{\lambda, \tilde{\nu}}\left(\theta_{0}\right)$ существует почти всюду. Действительно, из теоремы А следует, что для любого $0<q<1$

$\lim _{\alpha \rightarrow+\infty} \alpha \cdot m\left\{\theta \in\left(\theta_{0} ; \theta_{0}+\pi\right):\left|P\left(\tilde{\nu} ; q ; \theta_{0} ; \theta\right)\right|>\alpha\right\}=\sum_{n=1}^{\infty} \operatorname{ctg} \frac{\pi q^{n-1}}{2} \int_{\theta_{0}+\pi q^{n}}^{\theta_{0}+\pi q^{n-1}}\left|d \nu_{s}(\theta)\right|$ где $\nu_{s}-$ сингулярная часть меры $\nu$, и поэтому

$$
P_{1}\left(\tilde{\nu} ; \theta_{0}\right)=\int_{\theta_{0}}^{\theta_{0}+\pi} \operatorname{ctg} \frac{\theta-\theta_{0}}{2}\left|d \nu_{s}(\theta)\right|
$$

Аналогично,

$$
P_{2}\left(\tilde{\nu} ; \theta_{0}\right)=\int_{\theta_{0}-\pi}^{\theta_{0}} \operatorname{ctg} \frac{\theta_{0}-\theta}{2}\left|d \nu_{s}(\theta)\right| .
$$

Отсюда следует, что функции $P_{1}\left(\tilde{\nu} ; \theta_{0}\right)$ и $P_{2}\left(\tilde{\nu} ; \theta_{0}\right)$ и, следовательно, функция $r_{\lambda, \tilde{\nu}}\left(\theta_{0}\right)$ существуют почти всюду. Из $(12),(13)$ также следует, что функция $r_{\lambda, \tilde{\nu}}\left(\theta_{0}\right)$ не зависит от абсолютно непрерьвной части меры $\nu$. 
ТЕОРемА 3. Для любой меры $\nu \in M_{R}$ справедливо равенство

$$
\frac{2}{\pi} \int_{0}^{2 \pi} d \nu_{s}(\theta)=\lim _{\lambda \rightarrow+\infty} \lambda \int_{0}^{2 \pi} r_{\lambda, \tilde{\nu}}\left(\theta_{0}\right) d \theta_{0}
$$

Так как функция $r_{\lambda, \tilde{\nu}}\left(\theta_{0}\right)$ не зависит от абсолютно непрерывной части меры $\nu$, достаточно доказать теорему для сингулярных мер. Поэтому в дальнейшем в доказательстве этой теоремы будем считать меру $\nu$ сингулярной и в формуле (14) вместо $\nu_{s}$ будем писать $\nu$.

Приведем леммы, используемые при доказательстве теоремы 3.

ЛЕмма 1. Для любой меры $\nu \in M_{+}$справедливо равенство (14).

ДоКАЗАТЕЛЬСТво. Из равенств (12), (13) получим, что

$$
P_{1}\left(\tilde{\nu} ; \theta_{0}\right)=\int_{\theta_{0}}^{\theta_{0}+\pi} \operatorname{ctg} \frac{\theta-\theta_{0}}{2} d \nu(\theta), \quad P_{2}\left(\tilde{\nu} ; \theta_{0}\right)=\int_{\theta_{0}-\pi}^{\theta_{0}} \operatorname{ctg} \frac{\theta_{0}-\theta}{2} d \nu(\theta) .
$$

Следовательно, при $\tilde{\nu}\left(\theta_{0}\right)>\lambda(\lambda>0)$ имеем

$$
\begin{aligned}
r_{\lambda, \tilde{\nu}}\left(\theta_{0}\right) & =\operatorname{sign}\left(P_{2}\left(\tilde{\nu} ; \theta_{0}\right)-P_{1}\left(\tilde{\nu} ; \theta_{0}\right)\right) \\
& =\operatorname{sign}\left(\int_{\theta_{0}-\pi}^{\theta_{0}+\pi} \operatorname{ctg} \frac{\theta_{0}-\theta}{2} d \nu(\theta)\right)=\operatorname{sign} \tilde{\nu}\left(\theta_{0}\right)=1,
\end{aligned}
$$

а при $\tilde{\nu}\left(\theta_{0}\right)<-\lambda$ имеем $r_{\lambda, \tilde{\nu}}\left(\theta_{0}\right)=\operatorname{sign}\left(-\tilde{\nu}\left(\theta_{0}\right)\right)=1$.

Тогда

$\lim _{\lambda \rightarrow+\infty} \lambda \int_{0}^{2 \pi} r_{\lambda, \tilde{\nu}}\left(\theta_{0}\right) d \theta_{0}=\lim _{\lambda \rightarrow+\infty} \lambda m\left\{\theta_{0} \in T_{0}:\left|\tilde{\nu}\left(\theta_{0}\right)\right|>\lambda\right\}=\frac{2}{\pi}\left\|\nu_{s}\right\|=\frac{2}{\pi} \int_{0}^{2 \pi} d \nu_{s}(\theta)$.

Лемма доказана.

Лемма 2. Если мера $\nu_{1} \in M_{R}$ сосредоточена на множестве $A \subset T_{0}$, а мера $\nu_{2} \in M_{R}$ на $T_{0} \backslash A$, mo

$$
\lim _{\lambda \rightarrow+\infty} \lambda m\left(\left(\left|\tilde{\nu}_{1}\right|>\lambda\right) \cap\left(\left|\tilde{\nu}_{2}\right|>\lambda\right)\right)=0 .
$$

ДокаЗАТЕЛЬСтво. Возьмем любое число $\varepsilon>0$. Существуют меры $\mu_{1}, \mu_{2} \in M_{R} \mathrm{c}$ носителем соответственно в $A$ и $T \backslash A$ такие, что

$$
\left\|\mu_{1}-\nu_{1}\right\|<\frac{\varepsilon}{4}, \quad\left\|\mu_{2}-\nu_{2}\right\|<\frac{\varepsilon}{4} .
$$

Обозначим $F_{1}=\operatorname{supp} \mu_{1}, F_{2}=\operatorname{supp} \mu_{2}$. Для $\delta>0$ положим

$$
F_{j, \delta}=\left\{\theta \in T_{0}: \operatorname{dist}\left(\theta, F_{j}\right) \leqslant \delta\right\}, \quad j=1,2,
$$

где $\operatorname{dist}\left(\theta, F_{j}\right)$ обозначает евклидово расстояние от точки $\theta$ до множества $F_{j}$. Выберем $\delta>0$ настолько малым, что $F_{1, \delta} \cap F_{2, \delta}=\varnothing$. 
Обозначим

$$
\lambda_{\delta}=\max \left\{\sup _{\theta \in T_{0} \backslash F_{1, \delta}}\left|\tilde{\mu}_{1}(\theta)\right|, \sup _{\theta \in T_{0} \backslash F_{2, \delta}}\left|\tilde{\mu}_{2}(\theta)\right|\right\} .
$$

Возьмем $\lambda>2 \lambda_{\delta}$. Тогда из включений

$$
\left(\left|\tilde{\mu}_{1}\right|>\lambda\right) \subset F_{1, \delta}, \quad\left(\left|\tilde{\mu}_{2}\right|>\lambda\right) \subset F_{2, \delta}
$$

получим, что

$$
\left(\left|\tilde{\mu}_{1}\right|>\lambda\right) \cap\left(\left|\tilde{\mu}_{2}\right|>\lambda\right)=\varnothing .
$$

С другой стороны, из включений

$$
\begin{aligned}
& \left(\left|\tilde{\nu}_{1}\right|>\lambda\right) \subset\left(\left|\tilde{\mu}_{1}\right|>\frac{\lambda}{2}\right) \cup\left(\left|\tilde{\nu}_{1}-\tilde{\mu}_{1}\right|>\frac{\lambda}{2}\right), \\
& \left(\left|\tilde{\nu}_{2}\right|>\lambda\right) \subset\left(\left|\tilde{\mu}_{2}\right|>\frac{\lambda}{2}\right) \cup\left(\left|\tilde{\nu}_{2}-\tilde{\mu}_{2}\right|>\frac{\lambda}{2}\right)
\end{aligned}
$$

следует, что

$$
\left(\left|\tilde{\nu}_{1}\right|>\lambda\right) \cap\left(\left|\tilde{\nu}_{2}\right|>\lambda\right) \subset\left[\left(\left|\tilde{\mu}_{1}\right|>\frac{\lambda}{2}\right) \cap\left(\left|\tilde{\mu}_{2}\right|>\frac{\lambda}{2}\right)\right] \cup\left(\left|\tilde{\nu}_{1}-\tilde{\mu}_{1}\right|>\frac{\lambda}{2}\right) \cup\left(\left|\tilde{\nu}_{2}-\tilde{\mu}_{2}\right|>\frac{\lambda}{2}\right) .
$$

Отсюда

$$
\begin{aligned}
& \lim _{\lambda \rightarrow+\infty} \lambda m\left(\left(\left|\tilde{\nu}_{1}\right|>\lambda\right) \cap\left(\left|\tilde{\nu}_{2}\right|>\lambda\right)\right) \\
& \quad \leqslant \lim _{\lambda \rightarrow+\infty} \lambda m\left[\left(\left|\tilde{\mu}_{1}\right|>\frac{\lambda}{2}\right) \cap\left(\left|\tilde{\mu}_{2}\right|>\frac{\lambda}{2}\right)\right]+\lim _{\lambda \rightarrow+\infty} \lambda m\left(\left|\tilde{\nu}_{1}-\tilde{\mu}_{1}\right|>\frac{\lambda}{2}\right) \\
& \quad+\lim _{\lambda \rightarrow+\infty} \lambda m\left(\left|\tilde{\nu}_{2}-\tilde{\mu}_{2}\right|>\frac{\lambda}{2}\right) \\
& \quad=0+\frac{4}{\pi}\left\|\mu_{1, s}-\nu_{1, s}\right\|+\frac{4}{\pi}\left\|\mu_{2, s}-\nu_{2, s}\right\|<\varepsilon,
\end{aligned}
$$

где $\nu_{1, s}, \nu_{2, s}, \mu_{1, s}, \mu_{2, s}$ - сингулярные части соответственно мер $\nu_{1}, \nu_{2}, \mu_{1}, \mu_{2}$.

Из произвольности $\varepsilon$ получим равенство (15). Лемма доказана.

Теперь докажем теорему 3.

ДоКАЗАТЕЛЬСТво тЕоРЕмы 3. По теореме Хана-Жордана существуют неотрицательные меры $\nu_{1}$ и $\nu_{2}$, сосредоточенные соответственно на некоторых множествах $A$ и $T_{0} \backslash A$, такие, что $\nu=\nu_{1}-\nu_{2}$.

Тогда

$$
\begin{gathered}
P_{1}\left(\tilde{\nu} ; \theta_{0}\right)=\int_{\theta_{0}}^{\theta_{0}+\pi} \operatorname{ctg} \frac{\theta-\theta_{0}}{2}\left|d \nu_{1}(\theta)-d \nu_{2}(\theta)\right|=-\int_{\theta_{0}}^{\theta_{0}+\pi} \operatorname{ctg} \frac{\theta_{0}-\theta}{2}\left(d \nu_{1}(\theta)+d \nu_{2}(\theta)\right), \\
P_{2}\left(\tilde{\nu} ; \theta_{0}\right)=\int_{\theta_{0}-\pi}^{\theta_{0}} \operatorname{ctg} \frac{\theta_{0}-\theta}{2}\left(d \nu_{1}(\theta)+d \nu_{2}(\theta)\right) .
\end{gathered}
$$


Следовательно, при $\tilde{\nu}\left(\theta_{0}\right)>\lambda(\lambda>0)$

$$
\begin{aligned}
r_{\lambda, \tilde{\nu}}\left(\theta_{0}\right) & =\operatorname{sign}\left(P_{2}\left(\tilde{\nu} ; \theta_{0}\right)-P_{1}\left(\tilde{\nu} ; \theta_{0}\right)\right) \\
& =\operatorname{sign}\left(\int_{\theta_{0}-\pi}^{\theta_{0}+\pi} \operatorname{ctg} \frac{\theta_{0}-\theta}{2}\left(d \nu_{1}(\theta)+d \nu_{2}(\theta)\right)\right)=\operatorname{sign}\left(\tilde{\nu}_{1}\left(\theta_{0}\right)+\tilde{\nu}_{2}\left(\theta_{0}\right)\right),
\end{aligned}
$$

а при $\tilde{\nu}\left(\theta_{0}\right)<-\lambda r_{\lambda, \tilde{\nu}}\left(\theta_{0}\right)=-\operatorname{sign}\left(\tilde{\nu}_{1}\left(\theta_{0}\right)+\tilde{\nu}_{2}\left(\theta_{0}\right)\right)$.

Поэтому

$$
\lim _{\lambda \rightarrow+\infty} \lambda \int_{0}^{2 \pi} r_{\lambda, \tilde{\nu}}\left(\theta_{0}\right) d \theta_{0}=\lim _{\lambda \rightarrow+\infty} \lambda\left[J_{1}(\lambda)-J_{2}(\lambda)\right]
$$

где

$$
\begin{aligned}
& J_{1}(\lambda)=m\left((\tilde{\nu}>\lambda) \cap\left(\tilde{\nu}_{1}+\tilde{\nu}_{2}>0\right)\right)+m\left((\tilde{\nu}<-\lambda) \cap\left(\tilde{\nu}_{1}+\tilde{\nu}_{2}<0\right)\right) \\
& J_{2}(\lambda)=m\left((\tilde{\nu}>\lambda) \cap\left(\tilde{\nu}_{1}+\tilde{\nu}_{2}<0\right)\right)+m\left((\tilde{\nu}<-\lambda) \cap\left(\tilde{\nu}_{1}+\tilde{\nu}_{2}>0\right)\right) .
\end{aligned}
$$

Докажем, что

$$
\lim _{\lambda \rightarrow+\infty} \lambda J_{1}(\lambda)=\frac{2}{\pi} \int_{0}^{2 \pi} d \nu_{1}(\theta), \quad \lim _{\lambda \rightarrow+\infty} \lambda J_{2}(\lambda)=\frac{2}{\pi} \int_{0}^{2 \pi} d \nu_{2}(\theta) .
$$

Возьмем любое число $0<\varepsilon<1$.

Для любого $\theta \in(\tilde{\nu}>\lambda) \cap\left(\tilde{\nu}_{1}+\tilde{\nu}_{2}>0\right)$ из неравенств

$$
\tilde{\nu}(\theta)=\tilde{\nu}_{1}(\theta)-\tilde{\nu}_{2}(\theta)>\lambda, \quad \tilde{\nu}_{1}(\theta)+\tilde{\nu}_{2}(\theta)>0
$$

вытекает, что

$$
0<\tilde{\nu}_{1}(\theta)+\tilde{\nu}_{2}(\theta)<2 \tilde{\nu}_{1}(\theta)-\lambda .
$$

Отсюда $\tilde{\nu}_{1}(\theta)>\lambda / 2$. Поэтому

$$
\left((\tilde{\nu}>\lambda) \cap\left(\tilde{\nu}_{1}+\tilde{\nu}_{2}>0\right)\right) \subset\left(\left(\tilde{\nu}_{1}>(1-\varepsilon) \lambda\right) \cup\left[\left(\tilde{\nu}_{1}>\frac{\lambda}{2}\right) \cap\left(\tilde{\nu}_{2}<-\varepsilon \lambda\right)\right]\right) .
$$

Аналогично доказывается, что

$$
\left((\tilde{\nu}<-\lambda) \cap\left(\tilde{\nu}_{1}+\tilde{\nu}_{2}<0\right)\right) \subset\left(\left(\tilde{\nu}_{1}<-(1-\varepsilon) \lambda\right) \cup\left[\left(\tilde{\nu}_{1}<-\frac{\lambda}{2}\right) \cap\left(\tilde{\nu}_{2}>\varepsilon \lambda\right)\right]\right) .
$$

Из леммы 2 следует, что

$$
\begin{aligned}
\lim _{\lambda \rightarrow+\infty} \lambda J_{1}(\lambda) \leqslant & \lim _{\lambda \rightarrow+\infty} \lambda m\left(\left|\tilde{\nu}_{1}\right|>(1-\varepsilon) \lambda\right) \\
& +\lim _{\lambda \rightarrow+\infty} \lambda m\left[\left(\left|\tilde{\nu}_{1}\right|>\frac{\lambda}{2}\right) \cap\left(\left|\tilde{\nu}_{2}\right|>\varepsilon \lambda\right)\right]=\frac{2}{\pi} \cdot \frac{1}{1-\varepsilon}\left\|\nu_{1}\right\| .
\end{aligned}
$$

Отсюда из произвольности $\varepsilon>0$ получим, что

$$
\lim _{\lambda \rightarrow+\infty} \lambda J_{1}(\lambda) \leqslant \frac{2}{\pi}\left\|\nu_{1}\right\|=\frac{2}{\pi} \int_{0}^{2 \pi} d \nu_{1}(\theta)
$$


Аналогично доказывается, что

$$
\lim _{\lambda \rightarrow+\infty} \lambda J_{2}(\lambda) \leqslant \frac{2}{\pi} \int_{0}^{2 \pi} d \nu_{2}(\theta) .
$$

Следовательно,

$$
\lim _{\lambda \rightarrow+\infty} \lambda\left[J_{1}(\lambda)+J_{2}(\lambda)\right] \leqslant \frac{2}{\pi} \int_{0}^{2 \pi}\left[d \nu_{1}(\theta)+d \nu_{2}(\theta)\right]
$$

Но, с другой стороны,

$$
\begin{aligned}
\lim _{\lambda \rightarrow+\infty} \lambda\left[J_{1}(\lambda)+J_{2}(\lambda)\right] & =\lim _{\lambda \rightarrow+\infty} \lambda\left[m(|\tilde{\nu}|>\lambda)-m\left((|\tilde{\nu}|>\lambda) \cap\left(\tilde{\nu}_{1}+\tilde{\nu}_{2}=0\right)\right)\right] \\
& =\frac{2}{\pi}\|\tilde{\nu}\|-\lim _{\lambda \rightarrow+\infty} \lambda m\left(\left(\left|\tilde{\nu}_{1}-\tilde{\nu}_{2}\right|>\lambda\right) \cap\left(\tilde{\nu}_{2}=-\tilde{\nu}_{1}\right)\right) \\
& =\frac{2}{\pi}\|\tilde{\nu}\|-\lim _{\lambda \rightarrow+\infty} \lambda m\left(\left(2\left|\tilde{\nu}_{1}\right|>\lambda\right) \cap\left(\tilde{\nu}_{2}=-\tilde{\nu}_{1}\right)\right) \\
& =\frac{2}{\pi} \int_{0}^{2 \pi}\left[d \nu_{1}(\theta)+d \nu_{2}(\theta)\right]
\end{aligned}
$$

потому что

$$
\lim _{\lambda \rightarrow+\infty} \lambda m\left(\left(2\left|\tilde{\nu}_{1}\right|>\lambda\right) \cap\left(\tilde{\nu}_{2}=-\tilde{\nu}_{1}\right)\right) \leqslant \lim _{\lambda \rightarrow+\infty} \lambda m\left(\left(\left|\tilde{\nu}_{1}\right|>\frac{\lambda}{2}\right) \cap\left(\tilde{\nu}_{2}>\frac{\lambda}{2}\right)\right)=0 .
$$

Поэтому неравенства (18) и (19) превращаются в равенства, т.е. вьполняются равенства (17). Тогда из (16) получим, что

$$
\lim _{\lambda \rightarrow+\infty} \lambda \int_{0}^{2 \pi} r_{\lambda, \tilde{\nu}}\left(\theta_{0}\right) d \theta_{0}=\frac{2}{\pi} \int_{0}^{2 \pi}\left[d \nu_{1}(\theta)-d \nu_{2}(\theta)\right]=\frac{2}{\pi} \int_{0}^{2 \pi} d \nu(\theta) .
$$

Теорема доказана.

ЛЕмма 3. Для любой непрерывной по Гёльдеру функиии $f$ на $T_{0}$ и для любой меры $\nu \in M_{R}$ выполняется равенство

$$
\frac{2}{\pi} \int_{0}^{2 \pi} f(\theta) d \nu_{s}(\theta)=\lim _{\lambda \rightarrow+\infty} \lambda \int_{0}^{2 \pi} r_{\lambda, f \cdot \tilde{\nu}}\left(\theta_{0}\right) d \theta_{0}
$$

ДокАЗАТЕЛЬСтво. Рассмотрим меру $d \mu(\theta)=f(\theta) d \nu(\theta)$. Тогда

$$
\tilde{\mu}(\theta)=J(\theta)+f(\theta) \tilde{\nu}(\theta)
$$

где

$$
J(\theta)=\frac{1}{2 \pi} \int_{0}^{2 \pi} \operatorname{ctg} \frac{\theta-\theta_{0}}{2}\left[f\left(\theta_{0}\right)-f(\theta)\right] d \nu\left(\theta_{0}\right)
$$

- интегрируемая функция. 
Из теоремы 5 следует, что

$$
\frac{1}{\pi} \int_{0}^{2 \pi} f(\theta) d \nu_{s}(\theta)=\lim _{\lambda \rightarrow+\infty} \lambda \int_{0}^{2 \pi} r_{\lambda, \tilde{\mu}}\left(\theta_{0}\right) d \theta_{0} .
$$

Из интегрируемости функции $J(\theta)$ получим, что

$$
\lim _{\lambda \rightarrow+\infty} \lambda \cdot m(J>\lambda)=0
$$

и, следовательно (см. доказательство теоремы 1),

$$
\lim _{\lambda \rightarrow+\infty} \lambda \cdot m((|\tilde{\mu}|>\lambda) \Delta(|f \cdot \tilde{\nu}|>\lambda))=0 .
$$

Из (22) вытекает, что для любого $0<q<1$ пределы

$$
\lim _{\alpha \rightarrow+\infty} \alpha \cdot m\left\{\theta \in\left(\theta_{0} ; \theta_{0}+\pi\right):\left|P\left(\tilde{\mu} ; q ; \theta_{0} ; \theta\right)\right|>\alpha\right\}
$$

и

$$
\lim _{\alpha \rightarrow+\infty} \alpha \cdot m\left\{\theta \in\left(\theta_{0} ; \theta_{0}+\pi\right):\left|P\left(f \cdot \tilde{\nu} ; q ; \theta_{0} ; \theta\right)\right|>\alpha\right\}
$$

совпадают почти всюду. Тогда почти всюду

$$
P_{1}\left(\tilde{\mu} ; \theta_{0}\right)=P_{1}\left(f \cdot \tilde{\nu} ; \theta_{0}\right) .
$$

Аналогично доказывается, что почти всюду

$$
P_{2}\left(\tilde{\mu} ; \theta_{0}\right)=P_{2}\left(f \cdot \tilde{\nu} ; \theta_{0}\right) .
$$

Это означает, что почти для всех $\theta_{0} \in(|\tilde{\mu}|>\lambda) \cap(|f \cdot \tilde{\nu}|>\lambda)$

$$
r_{\lambda, \tilde{\mu}}\left(\theta_{0}\right)=r_{\lambda, f \cdot \tilde{\nu}}\left(\theta_{0}\right)
$$

Тогда

$$
\begin{aligned}
\int_{0}^{2 \pi} r_{\lambda, \tilde{\mu}}\left(\theta_{0}\right) d \theta_{0}= & \int_{(|\tilde{\mu}|>\lambda)} r_{\lambda, \tilde{\mu}}\left(\theta_{0}\right) d \theta_{0} \\
= & \int_{(|\tilde{\mu}|>\lambda) \cap(|f \cdot \tilde{\nu}|>\lambda)} r_{\lambda, f \cdot \tilde{\nu}}\left(\theta_{0}\right) d \theta_{0}+\int_{(|\tilde{\mu}|>\lambda) \backslash(|f \cdot \tilde{\nu}|>\lambda)} r_{\lambda, \tilde{\mu}}\left(\theta_{0}\right) d \theta_{0} \\
= & \int_{0}^{2 \pi} r_{\lambda, f \cdot \tilde{\nu}\left(\theta_{0}\right) d \theta_{0}-} \int_{(|f \cdot \tilde{\nu}|>\lambda) \backslash(|\tilde{\mu}|>\lambda)} r_{\lambda, f \cdot \tilde{\nu}}\left(\theta_{0}\right) d \theta_{0} \\
& +\int_{(|\tilde{\mu}|>\lambda) \backslash(|f \cdot \tilde{\nu}|>\lambda)} r_{\lambda, \tilde{\mu}}\left(\theta_{0}\right) d \theta_{0} .
\end{aligned}
$$

С другой стороны,

$$
\begin{aligned}
& \left|\int_{(|\tilde{\mu}|>\lambda) \backslash(|f \cdot \tilde{\nu}|>\lambda)} r_{\lambda, \tilde{\mu}}\left(\theta_{0}\right) d \theta_{0}-\int_{(|f \cdot \tilde{\nu}|>\lambda) \backslash(|\tilde{\mu}|>\lambda)} r_{\lambda, f \cdot \tilde{\nu}}\left(\theta_{0}\right) d \theta_{0}\right| \\
& \quad \leqslant m((|\tilde{\mu}|>\lambda) \Delta(|f \cdot \tilde{\nu}|>\lambda)) .
\end{aligned}
$$


Поэтому из (20) и (22) получим, что

$$
\frac{2}{\pi} \int_{0}^{2 \pi} f(\theta) d \nu_{s}(\theta)=\lim _{\lambda \rightarrow+\infty} \lambda \int_{0}^{2 \pi} r_{\lambda, \tilde{\mu}}\left(\theta_{0}\right) d \theta_{0}=\lim _{\lambda \rightarrow+\infty} \lambda \int_{0}^{2 \pi} r_{\lambda, f \cdot \tilde{\nu}}\left(\theta_{0}\right) d \theta_{0} .
$$

Лемма доказана.

Аналогично лемме 3 можно доказать, что для любых непрерьвных по Гёльдеру функций $f_{1}$ и $f_{2}$, и для любых мер $\nu_{1}, \nu_{2} \in M_{R}$ вьполняется равенство

$$
\frac{2}{\pi} \int_{0}^{2 \pi}\left(f_{1}(\theta) d \nu_{1, s}(\theta)+f_{2}(\theta) d \nu_{2, s}(\theta)\right)=\lim _{\lambda \rightarrow+\infty} \lambda \int_{0}^{2 \pi} r_{\lambda, f_{1} \cdot \tilde{\nu}_{1}+f_{2} \cdot \tilde{\nu}_{2}}\left(\theta_{0}\right) d \theta_{0},
$$

где $\nu_{1, s}$ и $\nu_{2, s}$ - сингулярные части соответственно мер $\nu_{1}$ и $\nu_{2}$.

В этом случае нужно рассмотреть меру $\mu=f_{1} \cdot \nu_{1}+f_{2} \cdot \nu_{2}$.

Лемма 4. Для любой меры $\nu \in M_{C}$ и непрерывной по Гёльдеру комплексной функиии $f=f_{1}+i f_{2}$ выполняется равенство

$\frac{2}{\pi} \int_{0}^{2 \pi} f(\theta) d \nu_{s}(\theta)=\lim _{\lambda \rightarrow+\infty} \lambda \int_{0}^{2 \pi} r_{\lambda, \operatorname{Re}(f \cdot \tilde{\nu})}\left(\theta_{0}\right) d \theta_{0}+i \lim _{\lambda \rightarrow+\infty} \lambda \int_{0}^{2 \pi} r_{\lambda, \operatorname{Im}(f \cdot \tilde{\nu})}\left(\theta_{0}\right) d \theta_{0}$.

ДокАЗАТЕЛЬСТво. Пусть $\nu=\nu_{1}+i \nu_{2}$. Тогда из равенства (24) получим, что

$$
\begin{aligned}
& \frac{2}{\pi} \int_{0}^{2 \pi} f(\theta) d \nu_{s}(\theta) \\
& \quad=\frac{2}{\pi} \int_{0}^{2 \pi}\left(f_{1}(\theta) d \nu_{1, s}(\theta)-f_{2}(\theta) d \nu_{2, s}(\theta)\right)+\frac{2 i}{\pi} \int_{0}^{2 \pi}\left(f_{1}(\theta) d \nu_{2, s}(\theta)+f_{2}(\theta) d \nu_{1, s}(\theta)\right) \\
& \quad=\lim _{\lambda \rightarrow+\infty} \lambda \int_{0}^{2 \pi} r_{\lambda, f_{1} \cdot \tilde{\nu}_{1}-f_{2} \cdot \tilde{\nu}_{2}}\left(\theta_{0}\right) d \theta_{0}+i \lim _{\lambda \rightarrow+\infty} \lambda \int_{0}^{2 \pi} r_{\lambda, f_{1} \cdot \tilde{\nu}_{2}+f_{2} \cdot \tilde{\nu}_{1}}\left(\theta_{0}\right) d \theta_{0} \\
& \quad=\lim _{\lambda \rightarrow+\infty} \lambda \int_{0}^{2 \pi} r_{\lambda, \operatorname{Re}(f \cdot \tilde{\nu})}\left(\theta_{0}\right) d \theta_{0}+i \lim _{\lambda \rightarrow+\infty} \lambda \int_{0}^{2 \pi} r_{\lambda, \operatorname{Im}(f \cdot \tilde{\nu})}\left(\theta_{0}\right) d \theta_{0} .
\end{aligned}
$$

Лемма доказана.

Теперь сформулируем основную теорему этой работы, т.е. представление аналитической функции через граничные значения для произвольных мер.

Теорема 4. Пусть мера $\nu \in M_{C}, u\left(r e^{i \varphi}\right)$ - ее интеграл Пуассона, a $v\left(r e^{i \varphi}\right)$ гармонически сопряженная функиия с и(rе $\left.e^{i \varphi}\right)$. Тогда

$$
\begin{gathered}
F(z)=\frac{1}{2 \pi i} \lim _{\lambda \rightarrow+\infty} \int_{T_{\lambda}} \frac{F(t)}{t-z} d t+\frac{1}{4 i} \lim _{\lambda \rightarrow+\infty} \lambda \int_{0}^{2 \pi} r_{\lambda, \operatorname{Re} h}\left(\theta_{0}\right) d \theta_{0} \\
+\frac{1}{4} \lim _{\lambda \rightarrow+\infty} \lambda \int_{0}^{2 \pi} r_{\lambda, \operatorname{Im} h}\left(\theta_{0}\right) d \theta_{0}, \quad z=r e^{i \varphi}
\end{gathered}
$$

где $h(\theta)=\frac{e^{i \theta}}{e^{i \theta}-z} F\left(e^{i \theta}\right), F(z)=u(z)+i v(z), F(t)-$ некасательные предельные значения функиии $F(z)$ при $z \rightarrow t=e^{i \theta}, T_{\lambda}=\left\{t=e^{i \theta}:|h(\theta)| \leqslant \lambda\right\}$. 
ДокАЗАТЕЛЬСтво. Обозначим $g(\theta)=i e^{i \theta} /\left(e^{i \theta}-z\right)$. Из леммы 4 вытекает, что

$$
\frac{2}{\pi} \int_{0}^{2 \pi} g(\theta) d \nu_{s}(\theta)=\lim _{\lambda \rightarrow+\infty} \lambda \int_{0}^{2 \pi} r_{\lambda, \operatorname{Re}(g \cdot \tilde{\nu})}\left(\theta_{0}\right) d \theta_{0}+i \lim _{\lambda \rightarrow+\infty} \lambda \int_{0}^{2 \pi} r_{\lambda, \operatorname{Im}(g \cdot \tilde{\nu})}\left(\theta_{0}\right) d \theta_{0} .
$$

Пусть $d \nu(\theta)=f(\theta) d \theta+d \nu_{s}(\theta)$. Тогда [9]

$$
F(t)=F\left(e^{i \theta}\right)=f(\theta)+i \tilde{\nu}(\theta)
$$

Отсюда

$$
g(\theta) \cdot \tilde{\nu}(\theta)=\frac{e^{i \theta}}{e^{i \theta}-z} \cdot i \tilde{\nu}(\theta)=\frac{e^{i \theta}}{e^{i \theta}-z}\left[F\left(e^{i \theta}\right)-f(\theta)\right]=h(\theta)+i g(\theta) f(\theta) .
$$

Так как функция $g(\theta) f(\theta)$ интегрируема, рассуждая как в доказательстве леммы 3 получим, что

$$
\begin{aligned}
\lim _{\lambda \rightarrow+\infty} \lambda \int_{0}^{2 \pi} r_{\lambda, \operatorname{Re}(g \cdot \tilde{\nu})}\left(\theta_{0}\right) d \theta_{0} & =\lim _{\lambda \rightarrow+\infty} \lambda \int_{0}^{2 \pi} r_{\lambda, \operatorname{Re} h}\left(\theta_{0}\right) d \theta_{0} \\
\lim _{\lambda \rightarrow+\infty} \lambda \int_{0}^{2 \pi} r_{\lambda, \operatorname{Im}(g \cdot \tilde{\nu})}\left(\theta_{0}\right) d \theta_{0} & =\lim _{\lambda \rightarrow+\infty} \lambda \int_{0}^{2 \pi} r_{\lambda, \operatorname{Im} h}\left(\theta_{0}\right) d \theta_{0} .
\end{aligned}
$$

Применяя лемму 4 к непрерывной по Гёльдеру функции $g(t)$, из равенств $(25),(26)$ и из теоремы 2 следует равенство (24).

Теорема доказана.

Автор выражает глубокую благодарность Т. С. Салимову за внимание к работе и полезное обсуждение.

\section{СПИСОК ЦИТИРОВАННОЙ ЛИТЕРАТУРЫ}

[1] Ульянов П. Л. Об $A$-интеграле Коши // УМН. 1956. Т. 11. № 5. С. 223-229.

[2] Виноградова И.А. Обобщенный интеграл и сопряженные функции // Матем. сб. 1976. Т. 99 (141). №1. С. $84-121$.

[3] Виноградова И. А. Об $L G^{*}$-интеграле типа Коши // Матем. заметки. 1976. Т. 20. № 5 . C. $645-653$.

[4] Riesz M. Sur les fonctions conjuguees // Math. Z. 1927. V. 27. P. 218-244.

[5] Ульянов П. Л. $A$-интеграл и сопряженные функции // Ученые зап. Моск. ун-та. 1956. Т. 181. C. $139-157$.

[6] Hruscev S. V., Vinogradov S. A. Free interpolation in the space of uniformly convergent Taylor series // Lect. Notes in Math. 1981. V. 864. P. 171-213.

[7] Салимов Т. С. К теореме Е. Титчмарша о сопряженной функции // Proceedings of A. Razmadze Math. Institute. 1993. V. 102. P. 99-114.

[8] Колмогоров А. Н., Фомин С. В. Элементы теории функций и функционального анализа. М.: Наука, 1989.

[9] Гарнетт Дж. Ограниченные аналитические функции. М.: Мир, 1984. 